TRANSACTIONS OF THE

AMERICAN MATHEMATICAL SOCIETY

Volume 365, Number 1, January 2013, Pages 491-509

S 0002-9947(2012)05624-8

Article electronically published on August 7, 2012

\title{
MÖBIUS ITERATED FUNCTION SYSTEMS
}

\author{
ANDREW VINCE
}

\begin{abstract}
Iterated function systems have been most extensively studied when the functions are affine transformations of Euclidean space and, more recently, projective transformations on real projective space. This paper investigates iterated function systems consisting of Möbius transformations on the extended complex plane or, equivalently, on the Riemann sphere. The main result is a characterization, in terms of topological, geometric, and dynamical properties, of Möbius iterated function systems that possess an attractor. The paper also includes results on the duality between the attractor and repeller of a Möbius iterated function system.
\end{abstract}

\section{INTRODUCTION}

Iterated function systems (IFSs) are a standard framework for describing and analyzing self-referential sets such as deterministic fractals [3, 9, 13] and some types of random fractals [5]. Most of the examples of iterated function systems in the literature consist of affine functions defined on Euclidean space [1]. Attractors of affine IFSs have many applications, including image compression [4, 10] and geometric modeling [7]. They relate to the theory of the joint spectral radius [6] and to wavelets 11. Recently, a rich theory has been developed for IFSs consisting of projective transformations defined on real projective space [2]. The intuition developed for affine IFSs concerning the behavior of attractors seems not to extend to the projective setting.

This paper concerns Möbius IFSs. A Möbius IFS consists of Möbius transformations on the extended complex plane, equivalently on the Riemann sphere, or equivalently consisting of complex projective transformations on the complex projective line. Iteration by the action of a group of Möbius transformations on the complex plane has been nicely explored, in a recreational, but mathematically serious, manner in [16.

The main result of this paper is a characterization of iterated function systems that possess an attractor. The characterization involves topological, geometric, and dynamical properties of the IFS as explained briefly in the paragraphs following the statement of the theorem below. The second theorem describes the duality between the attractor and repeller of a Möbius IFS. Figure1 shows the attractor and repeller,

Received by the editors April 8, 2011 and, in revised form, May 9, 2011.

2010 Mathematics Subject Classification. Primary 28A80.

Key words and phrases. Iterated function systems, Möbius transformation.

Thanks go to Michael Barnsley for always stimulating conversations on iterated function systems, and for graciously hosting my visit to the Australian National University, where much of this paper was written. 
on the Riemann sphere, of a Möbius IFS given in Example 7.4 of section 7 . In the theorem below, $\bar{X}$ denotes the closure of a subset $X$ of the extended complex plane $\widehat{\mathbb{C}}$.

Theorem 1.1. For a Möbius IFS F, the following conditions are equivalent:

(1) $F$ has an attractor $A \neq \widehat{\mathbb{C}}$,

(2) $F$ has a repeller $R \neq \widehat{\mathbb{C}}$,

(3) there is a nonempty open set $U \neq \widehat{\mathbb{C}}$ such that $F$ is topolgically contractive on $\bar{U}$,

(4) $F$ is contractive on an open set $U$ such that $\bar{U} \neq \widehat{\mathbb{C}}$,

(5) $\mathcal{R}(F) \neq \widehat{\mathbb{C}}$.

Moreover, a Möbius IFS can have at most one attractor.

Theorem 1.2. Assume that $F$ has an attractor $A \neq \widehat{\mathbb{C}}$. If $B_{A}$ is the basin of attraction and of $A$ and $B_{R}$ the basin of attraction of the corresponding repeller $R$, then $A \cap R=\emptyset$ and $B_{A}=\widehat{\mathbb{C}} \backslash R$ and $B_{R}=\widehat{\mathbb{C}} \backslash A$.

Basic definitions associated with the extended complex plane $\widehat{\mathbb{C}}$ and Möbius transformation on $\widehat{\mathbb{C}}$ are provided in section 2 . Three equivalent views of the action

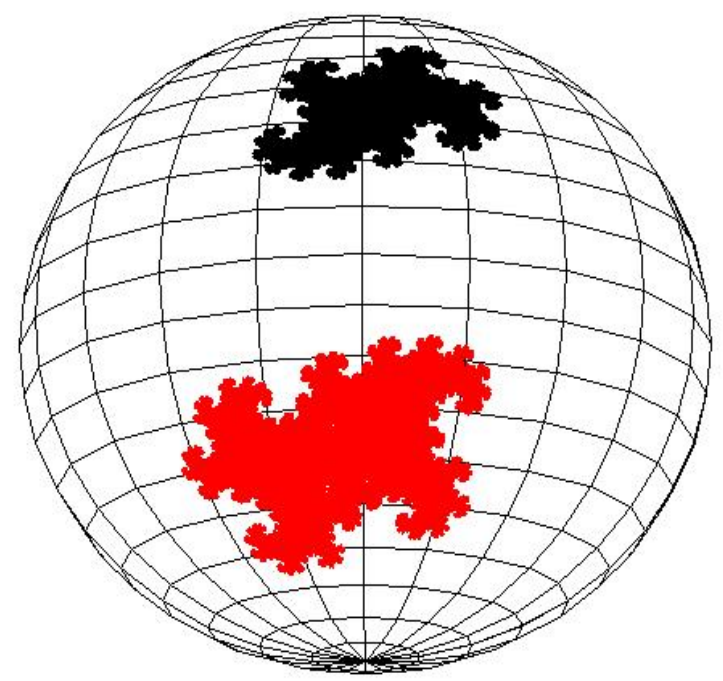

FiguRE 1. The attractor (red, below) and repeller (black, above) of a Möbius IFS consisting of two loxodromic transformations. See the online version for colors. 
of the Möbius group are described - as the action on the extended complex plane, as the action on the Riemann sphere via stereographic projection, and as the action of $\operatorname{PSL}(2, \mathbb{C})$ on the complex projective line. There is another viewpoint, as the action of the Lorentz group on the celestial sphere in Minkowski space. Although this action is not used in this paper, we describe it briefly in section 8 because of related papers in the literature on "quantum" iterated function systems.

The attractor of a Möbius IFS is a compact subset of $\widehat{\mathbb{C}}$, the definition and some properties of which are given in section 3. It is possible that the attractor of an IFS is all of $\widehat{\mathbb{C}}$; an example of a Möbius IFS with this property is given in that section. The definition and some properties of the repeller and the adjoint attractor are provided in section 5. The proof of the equivalence of statements (1) and (2) of Theorem 1.1 and the proof of Theorem 1.2 also appear in that section.

Concerning statements (3) and (4) in Theorem 1.1, a key issue is the relationship between the existence of an attractor and the contractive properties of the functions in the IFS. Topologically contractive in statement (3) means that each function in $F$ takes $\bar{U}$ into $U$. The proof of $(1) \Rightarrow(3)$ appears in section 3 , It is a classical result of Hutchinson 13 that, if an IFS $F$ consists of contractions on a complete metric space, then $F$ has an attractor. Contractive in statement (4) means that each function in $F$ is a contraction. Hence Hutchinson's result guarantees that $(4) \Rightarrow(1)$. It is also proved in section 3 that an IFS $F$ with an attractor $A \neq \widehat{\mathbb{C}}$ must be loxodromic, in the sense that all compositions of functions in $F$ must be loxodromic transformations. This is used to prove the uniqueness of the attractor as stated in Theorem 1.1.

The proof of $(3) \Rightarrow(4)$ appears in section 4 . What is somewhat subtle is that the metric with respect to which the functions in $F$ are contractions is not a standard metric. It is not the Euclidean metric on the complex plane $\mathbb{C}$ nor the chordal metric on the Riemann sphere. On the open set $U$ the metric is defined by

$$
d_{U}(x, y)=\max _{z \notin U} \log \frac{|z-x|}{|z-y|}+\max _{z \notin U} \log \frac{|z-y|}{|z-x|}
$$

if $x, y \in \mathbb{C}$. Examples of this metric for some sets $U$ are given in section 4. Figure 2 illustrates the formula above for the distance between two given points when $U$ is a disk.

The set $\mathcal{R}(F)$ of chain-recurrent points of an IFS $F$ is introduced in section 6 , The equivalence of statements (1) and (5) is proved in that section, which completes the proof of Theorem 1.1

Section 7 contains several examples of attractors of Möbius IFSs. The final section 9 poses an open problem concerning a property of a Möbius IFS that we call strong loxodromy. Strong loxodromy is defined in terms of the eigenvalues of the functions in the IFS. The conjecture is that $F$ is strongly loxodromic if and only if the five conditions in Theorem 1.1 hold.

\section{Extended complex plane, Riemann sphere, projective line}

This section contains basic notions related to three essentially equivalent spaces: the Riemann sphere $\mathbb{S}$, the extended complex plane $\widehat{\mathbb{C}}:=\mathbb{C} \cup\{\infty\}$, and the complex projective line $\mathbb{C} \mathbf{P}^{1}$. The complex projective line $\mathbb{C} \mathbf{P}^{1}$ is the quotient of $\mathbb{C}^{2} \backslash\{0\}$ by the equivalence relation $\left(z_{0}, z_{1}\right) \sim\left(\lambda z_{0}, \lambda z_{1}\right)$ for any nonzero $\lambda \in \mathbb{C}$. Let $\phi$ : $\mathbb{C}^{2} \rightarrow \mathbb{C} \mathbf{P}^{1}=\mathbb{C}^{2} / \sim$ denote the quotient map. The complex projective line $\mathbb{C} \mathbf{P}^{1}$ is 


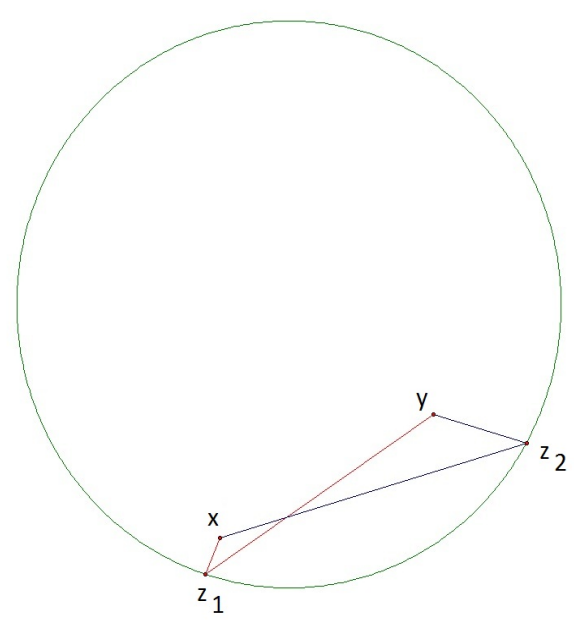

Figure 2. The distance between points $x$ and $y$ in the unit disk is approximately 3.18 . The point $z_{1}$ on the boundary of the disk maximizes the ratio $|z-y| /|z-x|$ of distances, and the point $z_{2}$ maximizes the ratio $|z-x| /|z-y|$ of distances.

topologically a sphere. The mapping

$$
\begin{aligned}
\mathbb{C P}^{1} & \rightarrow \widehat{\mathbb{C}}:=\mathbb{C} \cup\{\infty\}, \\
\left(z_{0}, z_{1}\right) & \mapsto \frac{z_{0}}{z_{1}}
\end{aligned}
$$

takes the complex projective line bijectively onto the extended complex plane, and the extended complex plane is in bijection with the Riemann sphere $\mathbb{S}$ via stereographic projection; explicitly

$$
\begin{aligned}
\mathbb{S} & \rightarrow \widehat{\mathbb{C}} \\
(x, y, z) & \mapsto \frac{x+i y}{1-z},
\end{aligned}
$$

where $\mathbb{S}$ is considered as the unit sphere centered at the origin in $\mathbb{R}^{3}$. Subsequently in this paper, we move interchangeably between $\mathbb{S}, \widehat{\mathbb{C}}$, and $\mathbb{C} \mathbf{P}^{1}$.

Denote by $|z-w|$ the Euclidean metric for $z, w \in \mathbb{C}$ and by $d_{c}(z, w)$ the chordal metric between $z, w \in \mathbb{S}$, where the points denoted $z$ and $w$ in $\mathbb{C}$ and in $\mathbb{S}$ are related by stereographic projection. If $z, w \in \mathbb{C}$ are contained in a disk of radius $R$ centered at the origin, then

$$
d_{c}(z, w) \leq 2|z-w| \leq\left(1+R^{2}\right) d_{c}(z, w),
$$

showing that the Euclidean metric and the chordal metric are Lipschitz equivalent on the disk and, in particular, induce the same topology there.

A dimension 1 projective transformation is an element of $P G L(2, \mathbb{C})=P S L(2, \mathbb{C})$, the quotient of the general linear group $G L(2, \mathbb{C})$ by the complex multiples of the identity matrix. Each element $f \in P S L(2, \mathbb{C})$ induces a well-defined map $f$ : $\mathbb{C} \mathbf{P}^{n} \rightarrow \mathbb{C P}^{n}$ such that, for any matrix $L$ representing $f$, the following diagram 
commutes:

$$
\begin{array}{ccc} 
& L & \\
\mathbb{C}^{2} & \rightarrow & \mathbb{C}^{2} \\
\phi \downarrow & & \downarrow \phi \\
\mathbb{C} \mathbf{P}^{1} & \rightarrow & \mathbb{C P}^{1} \\
& f &
\end{array}
$$

In particular, for any projective transformation $f: \mathbb{C} \mathbf{P}^{1} \rightarrow \mathbb{C} \mathbf{P}^{1}$, a corresponding linear map can be expressed as a $2 \times 2$ matrix $L_{f}=\left(\begin{array}{ll}a & b \\ c & d\end{array}\right)$ where $a d-b c=1$. Identifying the complex projective line $\mathbb{C P}^{1}$ with the extended complex plane $\widehat{\mathbb{C}}$, the group of projective transformations is the group of Möbius transformations of the form

$$
f(z)=\frac{a z+b}{c z+d}
$$

such that the complex numbers $a, b, c, d$ satisfy $a d-b c=1$ and with the understanding that $f(\infty)=a / c$ and $f(-d / c)=\infty$.

Definition 2.1. Letting $\operatorname{tr}(L)$ denote the trace of matrix $L$, a Möbius transformation $f$ is

(1) parabolic if $\operatorname{tr}^{2}\left(L_{f}\right)=4$,

(2) elliptic if $\operatorname{tr}\left(L_{f}\right)$ is real and $0 \leq \operatorname{tr}^{2}\left(L_{f}\right)<4$,

(3) loxodromic if $\operatorname{tr}^{2}\left(L_{f}\right) \notin[0,4]$.

A loxodromic transformation $f$ has two fixed points: an attractive fixed point denoted $\mathbf{z}_{\mathbf{f}}$ at which $\left|f^{\prime}\left(z_{f}\right)\right|<1$ and a repelling fixed point denoted $\mathbf{z}_{\mathbf{f}}^{\prime}$ at which $\left|f^{\prime}\left(z_{f}^{\prime}\right)\right|>1$.

\section{The ATtRACTOR OF A MÖBIUs IFS}

In this section, after giving the definition of an attractor of an IFS, the implication $(1) \Rightarrow(3)$ in Theorem 1.1 is proved. The notion of a loxodromic IFS is defined, and we prove that an IFS with an attractor $A \neq \widehat{\mathbb{C}}$ must be loxodromic.

Definition 3.1. Let $\mathbb{X}$ be a complete metric space. If $f_{m}: \mathbb{X} \rightarrow \mathbb{X}, m=1,2, \ldots, M$, are continuous mappings, then $F=\left(\mathbb{X} ; f_{1}, f_{2}, \ldots, f_{M}\right)$ is called an iterated function system (IFS). If $\mathbb{X}$ is $\widehat{\mathbb{C}}$ or $\mathbb{S}\left(\mathbb{C} \mathbf{P}^{1}\right)$ and each $f \in F$ is a Möbius transformation (projective transformation), then $F$ will be called a Möbius IFS.

To define the attractor of an IFS, first define

$$
F(B)=\bigcup_{f \in F} f(B)
$$

for any $B \subset \mathbb{X}$. By slight abuse of terminology, we use the same symbol $F$ for the IFS, the set of functions in the IFS, and for the above mapping. For $B \subset \mathbb{X}$, let $F^{k}(B)$ denote the $k$-fold composition of $F$, the union of $f_{i_{1}} \circ f_{i_{2}} \circ \cdots \circ f_{i_{k}}(B)$ over all finite words $i_{1} i_{2} \cdots i_{k}$ of length $k$. Define $F^{0}(B)=B$. Given a metric $d(\cdot, \cdot)$ on $\mathbb{X}$, there is a corresponding metric $d_{\mathbb{H}}$, called the Hausdorff metric, on the collection $\mathbb{H}(\mathbb{X})$ of all nonempty compact subsets of $\mathbb{X}$ :

$$
d_{\mathbb{H}}(B, C)=\max \left\{\sup _{b \in B} \inf _{c \in C} d(b, c), \sup _{c \in C} \inf _{b \in B} d(b, c)\right\} .
$$


Definition 3.2. A nonempty compact set $A \subset \mathbb{X}$ is said to be an attractor of the IFS $F$ if

(1) $F(A)=A$ and

(2) there is an open set $U \subset \mathbb{X}$ such that $A \subset U$ and $\lim _{k \rightarrow \infty} F^{k}(B)=A$, for all compact sets $B \subset U$, where the limit is with respect to the Hausdorff metric.

The largest open set $U$ such that (2) is true is called the basin of attraction for the attractor $A$ of the IFS $F$.

Example 3.3. It is possible that the attractor of a projective IFS on $\widehat{\mathbb{C}}$ is $\widehat{\mathbb{C}}$ itself. This is the case for $F=\left\{\widehat{\mathbb{C}} ; f_{0}, f_{1}, f_{2}, f_{3}, f_{4}, f_{5}\right\}$, where

$$
f_{0}(z)=z, \quad f_{1}(z)=z+1, \quad f_{2}=\frac{1}{z}, \quad f_{3}(z)=2 z, \quad f_{4}(z)=z / 3, \quad f_{5}(z)=e^{i \theta} z,
$$

where $\theta / \pi$ is irrational.

The first condition in Definition 3.2 of attractor holds, just by use of transformation $f_{0}$. Concerning the second condition in the definition of attractor, let $z_{0}$ be an arbitrary point in $\widehat{\mathbb{C}}$. The transformations $f_{1}$ and $f_{2}$ assure that $F\left(z_{0}\right)$ contains a point $w_{0} \neq 0, \infty$. Use the transformations $f_{0}, f_{3}$ and $f_{4}$ (and a little elementary number theory) to see that, for any $z$ on the line $\left\{z \mid \arg (z)=\arg \left(w_{0}\right)\right\}$ and any $\varepsilon>0$, there is a point $w \in F^{k}\left(w_{0}\right)$ such that $|w-z|<\varepsilon$ for $k$ sufficiently large. Hence there is a point $w \in F^{k}\left(z_{0}\right)$ such that $|w-z|<\varepsilon$ for $k$ sufficiently large. Finally, use the transformations $f_{0}$ and $f_{5}$ to guarantee that, for any $z \in \widehat{\mathbb{C}}$ and any $\varepsilon>0$, there is a point $w \in F^{k}\left(z_{0}\right)$ such that $|w-z|<\varepsilon$ for $k$ sufficiently large.

The notation $\operatorname{int}(Y)$ is used for the topological interior of a set $Y$ and $\bar{Y}$ for the closure of $Y$.

Definition 3.4. An IFS $F=\left(\mathbb{X} ; f_{1}, f_{2}, \ldots, f_{M}\right)$ is said to be topologically contractive on a compact set $K \subset \mathbb{X}$ if $F(K) \subset \operatorname{int}(K)$.

Although a similar result is used in the real projective case [2], we include it here for completeness.

Theorem 3.5. If a Möbius IFS $F$ on $\widehat{\mathbb{C}}$ has an attractor $A \neq \widehat{\mathbb{C}}$, then there exists an open set $U$ containing $A$ such that

(1) $A \subset U$,

(2) $\bar{U} \neq \widehat{\mathbb{C}}$,

(3) $F$ is topologically contractive on $\bar{U}$, and

(4) U has finitely many connected components.

Proof. Assume that the attractor $A \neq \widehat{\mathbb{C}}$; in particular there is a point $z_{0} \notin A$. Let $V$ be an open set such that $A \subset V$ and $\bar{V}$ contained in the basin of attraction for $A$. Let $V^{\prime}$ denote an open set such that $\bar{V} \subset V^{\prime}$ and $\overline{V^{\prime}} \subset \widehat{\mathbb{C}} \backslash\left\{z_{0}\right\}$. Since $A=\lim _{k \rightarrow \infty} F^{k}(\bar{V})$, there is an integer $m$ such that $F^{k}(\bar{V}) \subset V$ for all $k \geq m$. Define

$$
O:=\bigcup_{k=m}^{\infty} F^{k}(V) .
$$


Since each function in $F$ is an open map, $O$ has the properties:

(1) $O$ is open,

(2) $A \subset O$,

(3) $F^{k}(O) \subset V$ for all $k \geq 0$.

The next part of the proof makes use of the function $F^{-1}(X)=\{x \in \mathbb{X}: f(x) \in$ $X$ for all $f \in F\}$. Note that $F^{-1}$ takes open sets to open sets, $X \subset\left(F^{-1} \circ F\right)(X)$ and $\left(F \circ F^{-1}\right)(X) \subset X$ for all $X$.

Since $A=\lim _{k \rightarrow \infty} F^{k}(\bar{V})$, there is an integer $K$ such that $F^{K}(\bar{O}) \subset O$. Let $O_{k}, k=0,1, \ldots, K$, be defined recursively, going backwards from $O_{K}$ to $O_{0}$, as follows. Let $O_{K}=O$ and for $k=K-1, \ldots, 2,1,0$, let $O_{k}$ be an open set such that

(4) $F^{k}(\bar{O}) \subset O_{k} \subset V^{\prime}$, and

(5) $F\left(\bar{O}_{k}\right) \subset O_{k+1}$.

To verify that sets $O_{k}, k=K-1, \ldots, 1,0$, with these properties exist, first note that property (4) holds for $k=K$. To verify the properties for all $k=$ $K-1, \ldots, 2,1,0$, inductively assume that $O_{k}, k \geq 1$, satisfies property (4). Using property (4) we have $\mathcal{F}^{k-1}(\bar{O}) \subset \mathcal{F}^{-1}\left(\mathcal{F}^{k}(\bar{O})\right) \subset \mathcal{F}^{-1}\left(O_{k}\right)$, and using property (3) we have $\mathcal{F}^{k-1}(\bar{O}) \subset \bar{V} \subset V^{\prime}$. Now choose $O_{k-1}$ to be an open set such that $F^{k-1}(\bar{O}) \subset O_{k-1}$ and $\overline{O_{k-1}} \subset V^{\prime} \cap F^{-1}\left(O_{k}\right)$. The last inclusion implies $F\left(\bar{O}_{k-1}\right) \subset$ $O_{k}$.

We claim that

$$
U=\bigcup_{k=0}^{K-1} O_{k}
$$

satisfies the properties in the statement of the theorem. By properties (2) and (4) we have $A=\mathcal{F}^{k}(A) \subset \mathcal{F}^{k}(O) \subset O_{k}$ for each $k$, which implies $A \subset U$. By property (4) we have $U$ is an open set such that $\bar{U} \subset V^{\prime} \subset \widehat{\mathbb{C}} \backslash\left\{z_{0}\right\}$. Lastly, we show that $F$ is a topological contraction on $\bar{U}$ :

$$
\mathcal{F}(\bar{U}):=\bigcup_{k=0}^{K-1} \mathcal{F}\left(\overline{O_{k}}\right) \subset \bigcup_{k=1}^{K} O_{k}=\bigcup_{k=1}^{K-1} O_{k} \cup O_{K} \subset U \cup O \subset U \cup O_{0} \subset U,
$$

the first inclusion coming from property (5), the second inclusion because $O_{K}=O$, the third inclusion from property (4) applied to $k=0$, and the last inclusion from the definition of $U$.

Without loss of generality it may be assumed that each connected component $C$ of $U$ has nonempty intersection with $A$; otherwise, throw out the components that do not, and note that if $C$ has nonempty intersection with $A$, then so does $F(C)$. Since the components of $U$ form an open covering of $A$ and $A$ is compact, $U$ has finitely many components.

For an IFS $F$, let $\widehat{F}$ denote the set of all finite compositions of the functions in $F$. If each function in $\widehat{F}$ is loxodromic, then $F$ will be called a loxodromic IFS.

Theorem 3.6. If a Möbius IFS $F$ on $\widehat{\mathbb{C}}$ has an attractor $A \neq \widehat{\mathbb{C}}$ and $f \in F$, then

(1) $F$ is loxodromic,

(2) the attractive fixed point of each $f \in \widehat{F}$ lies in $A$, and

(3) the repulsive fixed point of each $f \in \widehat{F}$ lies outside the basin of attraction of $A$. 
Proof. Let $f \in \widehat{F}$ and $F^{\prime}=F \cup\{f\}$. It is routine to check that if $A$ is an attractor of $F$ with basin of attraction $B$, then $A$ is also an attractor of $F^{\prime}$ with basin of attraction $B$. Therefore, to prove statement (1) it is sufficient, without loss of generality, to show that each $f \in F$ is loxodromic, and to prove statements (2) and (3), we can, without loss of generality, assume that $f \in F$.

We will prove, by contradiction, that $F$ can contain no parabolic or elliptic transformation. Assume that $F$ has attractor $A^{\prime} \neq \widehat{\mathbb{C}}$ and that $F$ contains a parabolic transformation $f: \widehat{\mathbb{C}} \rightarrow \widehat{\mathbb{C}}$. Any parabolic transformation is conjugate (via a Möbius transformation $h$ ) to a translation of the form $g(z)=h f h^{-1}(z)=$ $z+1$. Let $F_{h}$ be the IFS obtained from $F$ by conjugating each of its functions by $h$. Then $F_{h}$ has attractor $A=h\left(A^{\prime}\right) \neq \widehat{\mathbb{C}}$. The point $\infty \in A$ because, if $z_{0}$ is any point in the basin of attraction of $A$, then $\infty=\lim _{k \rightarrow \infty} g^{n}\left(z_{0}\right) \in A$. Therefore, according to Theorem 3.5. there is a compact set $K:=\bar{U} \neq \widehat{\mathbb{C}}$ such that $\infty \in A \subset \operatorname{int}(K)$ and $F_{h}$ is a topological contraction on $K$. Since $\infty \in \operatorname{int}(K)$, there is some disk $D$ centered at the origin in $\mathbb{C}$ such that $K$ contains the complement of $D$. If $z$ is any point not in $K$, then $z-k \in D \subset K$ for $k$ sufficiently large. This implies that, for $k$ sufficiently large, $g^{k}(z-k)=z$ where $z-k \in K$ but $z \notin K$, contradicting that $F_{h}$ is a topological contraction.

Next assume that $F$ has attractor $A^{\prime} \neq \widehat{\mathbb{C}}$ and that $F$ contains an elliptic transformation $f$. Any elliptic transformation is conjugate (via a projective transformation $h$ ) to a projective transformation of the form $g(z)=h f h^{-1}(z)=\left(\begin{array}{cc}e^{i \theta} & 0 \\ 0 & e^{-i \theta}\end{array}\right)$, which, on the Riemann sphere centered at the origin in $\mathbb{R}^{3}$, is a rotation through the $x_{3}$-axis. By Theorem 3.5 there is a compact set $K$ that is mapped by $f$ into $\operatorname{int}(K)$. Therefore $g$ maps $h(K)$ into $\operatorname{int}(h(K))$. Since a rotation is an isometry of the sphere, this is impossible.

By statement (1) of this theorem, the map $f$ is loxodromic. Therefore $f$ has an attractive fixed point $z_{f}$ and a repulsive fixed point $z_{f}^{\prime}$. Moreover $\lim _{n \rightarrow \infty} f^{n}(z)=$ $z_{f}$ for any $z \neq z_{f}^{\prime}$. If $z$ is any point in the basin of attraction of $F$ that is not a fixed point of $f$, then $z_{f}=\lim _{k \rightarrow \infty} f^{k}(z) \subset \lim _{k \rightarrow \infty} F^{k}(z)=A$.

Corollary 3.7. A Möbius IFS can have at most one attractor.

Proof. Assume, by way of contradiction, that $F$ has two attractors, $A$ and $A^{\prime}$. First consider the case where $A \cap A^{\prime} \neq \emptyset$. If $z \in A \cap A^{\prime}$, then $A=\lim _{k \rightarrow \infty} F^{k}(\{z\})=A^{\prime}$. Therefore $A \cap A^{\prime}=\emptyset$. This implies that $A \neq \widehat{\mathbb{C}}$ and $A^{\prime} \neq \widehat{\mathbb{C}}$. By Theorem 3.6. the attractive fixed point of each $f \in F$ would lie in both $A$ and $A^{\prime}$, contradicting $A \cap A^{\prime}=\emptyset$.

\section{A metric ON SUbSETS OF $\widehat{\mathbb{C}}$}

This section concerns the contractive properties of a Möbius IFS. A metric is defined on any open set $U \subset \widehat{\mathbb{C}}$ with $\bar{U} \neq \widehat{\mathbb{C}}$. Using this metric, the implication $(3) \rightarrow(4)$ in Theorem 1.1 is proved.

Definition 4.1. A function $f: \mathbb{X} \rightarrow \mathbb{X}$ on a metric space $\mathbb{X}$ is called a contraction with respect to a metric $d$ if there is an $s, 0 \leq s<1$, such that $d(f(x), f(y)) \leq$ $s d(x, y)$ for all $x, y \in \mathbb{X}$. An IFS $F=\left(\mathbb{X} ; f_{1}, f_{2}, \ldots, f_{M}\right)$ is said to be contractive on a set $X \subset \mathbb{X}$ if

(1) $F(X) \subset X$, 
(2) there is a metric $d: X \times X \rightarrow[0, \infty)$, inducing the same topology as on $X$

(3) $(X, d)$ is a complete metric space, and

(4) for each $f \in F$, the restriction $\left.f\right|_{X}$ of $f$ to $X$ is a contraction on $X$ with respect to $d$.

It is a standard result that $F$ contractive on $X$ implies that the function $F$ : $\mathbb{H}(X) \rightarrow \mathbb{H}(X)$ is a contraction with respect to the corresponding Hausdorff metric.

Let $U$ be an open subset of $\widehat{\mathbb{C}}$ such that $\bar{U} \neq \widehat{\mathbb{C}}$. Define a function $d_{U}: U \times U \rightarrow$ $[0, \infty)$ by

$$
d_{U}(x, y)=\max _{z \notin U} \log \frac{|z-x|}{|z-y|}+\max _{z \notin U} \log \frac{|z-y|}{|z-x|}
$$

if $x, y \in \mathbb{C}$,

$$
d_{U}(x, \infty)=\max _{z \notin U} \log |z-x|+\max _{z \notin U} \log \frac{1}{|z-x|},
$$

and $d_{U}(\infty, y)$ is defined by an analogous formula. We claim that $d_{U}$ is a metric on $U$. Clearly $d_{U}(x, y)=d_{U}(y, x)$, and the triangle inequality is easy to check. Also $d_{U}(x, x)=0$ for all $x \in U$, and if $d_{U}(x, y)=0$, then $|z-x|=|z-y|$ for every $z \notin U$. Since $\bar{U} \neq \widehat{\mathbb{C}}$, this is possible only if $x=y$.

Lemma 4.2. Let $c>0$ be a real number, $x, y \in \mathbb{C}$, and $f$ a Möbius transformation.

(1) The locus of points $C=\left\{z \in \mathbb{C}: \frac{|z-x|}{|z-y|}=c\right\}$ is a circle with center on the line joining $x$ and $y$ if $c \neq 1$. If $c=1$, then $C$ is the perpendicular bisector of the line segment $\overline{x y}$.

(2) If $c>1$, then the set of points inside $C$ is $\left\{z \in \mathbb{C}: \frac{|z-x|}{|z-y|}>c\right\}$.

(3) There is a $c^{\prime}>0$ such that $f(C)=\left\{z \in \mathbb{C}: \frac{|z-f(x)|}{|z-f(y)|}=c^{\prime}\right\}$.

Proof. A straightforward calculation suffices to vertify statements (1) and (2). To vertify (3), it is sufficient to show that, if $z_{1}$ and $z_{2}$ are any points on $C$ such that $\frac{\left|z_{1}-x\right|}{\left|z_{1}-y\right|}=\frac{\left|z_{2}-x\right|}{\left|z_{2}-y\right|}$, then $\frac{\left|f\left(z_{1}\right)-f(x)\right|}{\left|f\left(z_{1}\right)-f(y)\right|}=\frac{\left|f\left(z_{2}\right)-f(x)\right|}{\left|f\left(z_{2}\right)-f(y)\right|}$. But this follows from the invariance of the cross ratio under the Möbius transformation $f$, i.e.,

$$
\frac{\left(z_{1}-x\right)\left(z_{2}-y\right)}{\left(z_{1}-y\right)\left(z_{2}-x\right)}=\frac{\left(f\left(z_{1}\right)-f(x)\right)\left(f\left(z_{2}\right)-f(y)\right)}{\left(f\left(z_{1}\right)-f(y)\right)\left(f\left(z_{2}\right)-f(x)\right)} .
$$

In order to generalize distance in the Cayley-Klein disk model of the real projective plane, Hilbert [12] defined a certain metric on convex cones in $\mathbb{R}^{n}$, now referred to as the Hilbert metric. The following examples make it clear that the metric defined by (4.1) and (4.2) is not this Hilbert metric, not even for a disk or a rectangle. However, as is the case for the Hilbert metric, the cross ratio plays an important role for the metric $d_{U}$, as is made clear in the proof of Lemma 4.2 above and Theorem 4.5 below.

Example 4.3. Let $U$ be the open unit disk in $\mathbb{C}$. The distance in the metric $d_{U}$ between the center of the disk and a point at a distance $r$ from the center is $\log \frac{1+r}{1-r}$. A general formula for the distance between two arbitrary points in $U$ is problematic because it involves solving a degree 6 polynomial equation. In general, the points $z$ that maximize in formula (4.1) do not lie on the line joining the two points. This 
is illustrated in Figure 2, Even for the disk, the metric $d_{U}$ is not the Hilbert metric associated with a 2-dimensional convex region.

Example 4.4. Let $U$ be the open rectangle in $\mathbb{C}$ bounded by the lines $\operatorname{Re}(z)=a+b, \operatorname{Re}(z)=-(a+b), \operatorname{Im}(z)=1, \operatorname{Im}(z)=-1$, where $a \geq 1, b \geq \sqrt{2}-1$. A staightforward calculation shows that $d_{U}(a,-a)=\log \left(a^{2}+1+a \sqrt{a^{2}+1}\right)-$ $\log \left(a^{2}+1-a \sqrt{a^{2}+1}\right)$ and the points that maximize the two quantities in formula (4.1) are $z_{1}=\sqrt{a^{2}+1} \pm i$ and $z_{2}=-\sqrt{a^{2}+1} \pm i$.

Theorem 4.5. Let $U$ be an open subset of $\widehat{\mathbb{C}}$ such that $\bar{U} \neq \widehat{\mathbb{C}}$.

(1) The metrics $d_{U}, d_{c}$ and the Euclidean metric induce the same topology on $U$.

(2) The metric space $\left(U, d_{U}\right)$ is complete.

(3) If $f: U \rightarrow U$ is a Möbius transformation such that $f(\bar{U}) \subset U$, then $f$ is a contraction with respect to $d_{U}$.

Proof. Concerning statement (1) in the theorem, the equivalence of the Euclidean and chordal metrics was already mentioned in section 2. The equivalence to the metric $d_{U}$ is left as a routine exercise. Concerning statement (2), assume that $\left\{z_{n}\right\}_{n=1}^{\infty}$ is a Cauchy sequence of points in $U$ with respect to $d_{U}$. By the definition of $d_{U}$, this implies that there is an $a>0$ such that $\left|z_{n}-z\right| \geq a$ for all $z \notin U$ and all $n$ sufficiently large. This in turn implies that $\left\{z_{n}\right\}_{n=1}^{\infty}$ is a Cauchy sequence with respect to the Euclidean metric, and hence a convergent sequence with respect to the Euclidean metric since the Euclidean metric is complete on $\bar{U}$. Since the Euclidean metric and the metric $d_{U}$ induce the same topology on $U$, the sequence $\left\{z_{n}\right\}_{n=1}^{\infty}$ is also convergent with respect to $d_{U}$.

Concerning statement (3), let $f$ be such a transformation. Let $x$ and $y$ be any two distinct points contained in $U$, neither equal to $\infty$. For $x=\infty$ or $y=\infty$, we omit the proof since it is similar but easier. We will show the existence of a real number $s, 0 \leq s<1$, such that $d_{U}(f(x), f(y)) \leq s d_{U}(x, y)$. Let $z_{1}$ and $z_{2}$ be arbitrary points not in $U$. The invariance of the cross ratio (equation (4.3)) implies

$$
\log \left|\frac{z_{1}-x}{z_{1}-y}\right|+\log \left|\frac{z_{2}-y}{z_{2}-x}\right|=\log \left|\frac{f\left(z_{1}\right)-f(x)}{f\left(z_{1}\right)-f(y)}\right|+\log \left|\frac{f\left(z_{2}\right)-f(y)}{f\left(z_{2}\right)-f(x)}\right|
$$

Let $w_{1}$ and $w_{2}$ be such that $\left|\frac{w_{1}-x}{w_{1}-y}\right|=\max _{z \notin U}\left|\frac{z-x}{z-y}\right|$ and $\left|\frac{w_{2}-y}{w_{2}-x}\right|=\max _{z \notin U}\left|\frac{z-y}{z-x}\right|$. From Lemma 4.2 it follows that $w_{1}$ and $w_{2}$ lie on the boundary of $U$, and the sets $D_{1}:=\left\{w:\left|\frac{w-x}{w-y}\right| \geq \max _{z \notin U}\left|\frac{z-x}{z-y}\right|\right\}$ and $D_{2}:=\left\{w:\left|\frac{w-y}{w-x}\right| \geq \max _{z \notin U}\left|\frac{z-y}{z-x}\right|\right\}$ are closed disks contained in $\bar{U}$. A point $w \in D_{1}$ such that $w \notin U$ would contradict the definition of $w_{1}$ as the point that maximizes $\left|\frac{z-x}{z-y}\right|$ over all $z \notin U$. The same is true for point $w \in D_{2}, w \notin U$. Moreover, by Lemma $4.2 f\left(D_{1}\right)=$ $\left\{z:\left|\frac{z-f(x)}{z-f(y)}\right| \geq\left|\frac{f\left(w_{1}\right)-f(x)}{f\left(w_{1}\right)-f(y)}\right|\right\}$. Since $f(\bar{U}) \subset U$, we have $f\left(D_{1}\right) \subset U$. Therefore there is an $s_{1}<1$ such that, if $z \notin U$, then $\left|\frac{z-f(x)}{z-f(y)}\right| \leq\left|\frac{f\left(w_{1}\right)-f(x)}{f\left(w_{1}\right)-f(y)}\right|^{s_{1}}$. Similarly, there is an $s_{2}<1$ such that, if $z \notin U$, then $\left|\frac{z-f(y)}{z-f(x)}\right| \leq\left|\frac{f\left(w_{2}\right)-f(y)}{f\left(w_{2}\right)-f(x)}\right|^{s_{2}}$. If 
$s=\max \left\{s_{1}, s_{2}\right\}$, then

$$
\begin{aligned}
d_{U}(f(x), f(y)) & =\max _{z \notin U} \log \left|\frac{z-f(x)}{z-f(y)}\right|+\max _{z \notin U} \log \left|\frac{z-f(y)}{z-f(x)}\right| \\
& \leq s\left(\log \left|\frac{f\left(w_{1}\right)-f(x)}{f\left(w_{1}\right)-f(y)}\right|+\log \left|\frac{f\left(w_{2}\right)-f(y)}{f\left(w_{2}\right)-f(x)}\right|\right) \\
& =s\left(\log \left|\frac{w_{1}-x}{w_{1}-y}\right|+\log \left|\frac{w_{2}-y}{w_{2}-x}\right|\right) \\
& =s\left(\max _{z \notin U} \log \left|\frac{z-x}{z-y}\right|+\max _{z \notin U} \log \left|\frac{z-y}{z-x}\right|\right)=s d_{U}(x, y) .
\end{aligned}
$$

The second equality is from equation (4.4).

The implication $(3) \Rightarrow(4)$ in the Theorem 1.1 is a direct consequence of Theorem 4.5 .

Corollary 4.6. For a Möbius IFS $F$, if there is an open set $U \neq \widehat{\mathbb{C}}$ such that $F$ is topologically contractive on $\bar{U}$, then $F$ is contractive on $U$.

\section{The ADJOINT ATtractor AND The REPELleR}

The adjoint and the repeller of a Möbius IFS are introduced in this section, and the equivalence of statements (1) and (2) in Theorem 1.1 is proved. The following notation is used. For $z \in \widehat{\mathbb{C}}$, denote the conjugate by $\bar{z}$, and for any $X \subset \widehat{\mathbb{C}}$, let

$$
\begin{aligned}
z^{\perp} & :=-1 / \bar{z}, \\
X^{\perp} & :=\left\{z^{\perp}: z \in X\right\}, \\
X^{*} & :=\widehat{\mathbb{C}} \backslash X^{\perp} .
\end{aligned}
$$

We use the notation $\perp: X \mapsto X^{\perp}$ and $*: X \mapsto X^{*}$ for the corresponding mappings. Identifying $\widehat{\mathbb{C}}$ with $\mathbb{C} \mathbf{P}^{1}$, we have

$$
z^{\perp}=\{w:\langle w, z\rangle=0\} .
$$

If $F$ is a Möbius IFS and $f \in F$, then its inverse $f^{-1}$ is represented by the matrix $L_{f^{-1}}:=L_{f}^{-1}$. In a similar fashion, define $f^{*}$ and $f^{-*}$ as the Möbius transformations represented by

$$
L_{f^{*}}:=L_{f}^{*} \quad \text { and } \quad L_{f^{-*}}:=\left(L_{f}^{-1}\right)^{*}=\left(L_{f}^{*}\right)^{-1},
$$

respectively, where $*$ denotes the conjugate transpose matrix. The following iterated function systems related to $F$ will be used in this section.

(1) The adjoint of the IFS F, denoted by $F^{*}$, is the IFS

$$
F^{*}:=\left(\widehat{\mathbb{C}} ; f_{1}^{*}, f_{2}^{*}, \ldots, f_{M}^{*}\right) .
$$

(2) The inverse of the IFS F is the IFS

$$
F^{-1}:=\left(\widehat{\mathbb{C}} ; f_{1}^{-1}, f_{2}^{-1}, \ldots, f_{M}^{-1}\right) .
$$

Definition 5.1. A set $R \subset \widehat{\mathbb{C}}$ is said to be a repeller of the IFS $F$ if $R$ is the attractor of $F^{-1}$. A set $A^{\prime} \subset \widehat{\mathbb{C}}$ is said to be an adjoint attractor of the IFS $F$ if $A^{\prime}$ is the attractor of $F^{*}$. 
Proposition 5.2. The following diagrams commute for any Möbius transformation $f$ and any Möbius IFS F:

$$
\begin{array}{cccccc}
\widehat{C} & \perp & & & \perp & \\
f^{t} \downarrow & & \widehat{\mathbb{C}} & \mathbb{H}(\widehat{\mathbb{C}}) & \rightarrow & \mathbb{H}(\widehat{\mathbb{C}}) \\
\widehat{\mathbb{C}} & \rightarrow & f^{-1} & F^{*} \downarrow & & \downarrow F^{-1} \\
& \perp & & \mathbb{C}(\widehat{\mathbb{C}}) & \rightarrow & \mathbb{H}(\widehat{\mathbb{C}}) . \\
& & & \perp &
\end{array}
$$

Proof. To verify that the diagrams commute, it is sufficient to show that, for all $x \in \mathbb{C} \mathbf{P}^{1}$ and any Möbius transformation $f$, we have $L_{f}^{-1}\left(x^{\perp}\right)=\left[L_{f}^{t}(x)\right]^{\perp}$. But

$$
\begin{aligned}
L_{f}^{-1}\left(x^{\perp}\right) & =\left\{L_{f}^{-1} y:\langle x, y\rangle=0\right\}=\left\{z:\left\langle x, L_{f} z\right\rangle=0\right\} \\
& =\left\{z:\left\langle L_{f}^{t} x, z\right\rangle=0\right\}=\left[L_{f}^{t}(x)\right]^{\perp} .
\end{aligned}
$$

Let $\mathbb{S}(\widehat{\mathbb{C}})$ denote the set of all subsets of $\widehat{\mathbb{C}}$ (including the empty set). For an IFS $F$ define the operator $\bar{F}: \mathbb{S}(\widehat{\mathbb{C}}) \rightarrow \mathbb{S}(\widehat{\mathbb{C}})$ by

$$
\bar{F}(X)=\bigcap_{f \in F} f^{-*}(X)
$$

for any $X \in \mathbb{S}(\widehat{\mathbb{C}})$.

Proposition 5.3. The map * is an inclusion reversing function with these properties:

(1) The following diagram commutes:

$$
\begin{array}{ccc} 
& * & \\
\mathbb{S}(\widehat{\mathbb{C}}) & \rightarrow & \mathbb{S}(\widehat{\mathbb{C}}) \\
F \downarrow & & \downarrow \bar{F} \\
\mathbb{S}(\widehat{\mathbb{C}}) & \rightarrow & \mathbb{S}(\widehat{\mathbb{C}}) .
\end{array}
$$

(2) If $F(X) \subset Y$, then $F^{*}\left(Y^{*}\right) \subset X^{*}$.

Proof. The fact that the diagrams commute is easy to verify. Concerning the second statement, since $*$ is inclusion reversing, $F(X) \subset Y$ implies that $Y^{*} \subset[F(X)]^{*}=$ $\bar{F}\left(X^{*}\right)$, the equality coming from the commuting diagram. The definition of $\bar{F}$ then yields $F^{*}\left(Y^{*}\right) \subset X^{*}$.

Theorem 5.4. For a Möbius IFS $F$ the following statements are equivalent.

(1) $F$ has an attractor $A \neq \widehat{\mathbb{C}}$,

(2) $F^{*}$ has an adjoint attractor $A^{\prime} \neq \widehat{\mathbb{C}}$,

(3) $F$ has a repeller $R=\left(A^{\prime}\right)^{\perp} \neq \widehat{\mathbb{C}}$.

Moreover, if $B_{A}, B_{A^{\prime}}$, and $B_{R}$ are the basins of attraction for $A, A^{\prime}$, and $R$, of IFSs $F, F^{*}$ and $F^{-1}$, respectively, then

(1) $B_{R}=\widehat{\mathbb{C}} \backslash A$ and $B_{A}=\widehat{\mathbb{C}} \backslash R$, and

(2) $B_{A^{\prime}}=(\widehat{\mathbb{C}} \backslash A)^{\perp}$.

Proof. (1) $\Rightarrow(2)$ : At this point we have aleady proved the equivalence of statements (1) and (3) in Theorem 1.1. Hence if $F$ has an attractor, then there is a nonempty open set $U$ such that $F(\bar{U}) \subset U$ and $\bar{U} \neq \widehat{\mathbb{C}}$. By Proposition 5.3 this implies that 
$F^{*}\left(U^{*}\right) \subset(\bar{U})^{*}$. Let $V=(\bar{U})^{*}$. It is easy to check that $V=\emptyset$ if and only if $\bar{U}=\widehat{\mathbb{C}}$ and $\bar{V}=\widehat{\mathbb{C}}$ if and only if $U=\emptyset$; also routine is that $\bar{V}=U^{*}$, which is an open set. We now have $F^{*}(\bar{V})=V$, where $V$ is a nonempty open set and $\bar{V} \neq \widehat{\mathbb{C}}$. By the already proved implication $(3) \Rightarrow(1)$ in Theorem 1.1. the IFS $F^{*}$ has an attractor, say $A^{\prime} \neq \widehat{\mathbb{C}}$.

$(2) \Rightarrow(3)$ : To show that $A^{\perp \perp}$ is an attractor of $F^{-1}$, consider the first of the two conditions in the definition of an attractor. From the commuting diagram in Proposition 5.2, we have $F^{*}\left(A^{\prime}\right)=A^{\prime}$ if and only if $F^{-1}\left(A^{\prime \perp}\right)=\left(F^{*}\left(A^{\prime}\right)\right)^{\perp}=A^{\prime \perp}$. Concerning the second of the two conditions in the definition of an attractor, let $B$ be an arbitrary subset contained in the basin of attraction for $A^{\prime}$ of $F^{*}$. With respect to the Hausdorff metric, $\lim _{k \rightarrow \infty} F^{* k}(B)=A^{\prime}$ if and only if

$$
\lim _{k \rightarrow \infty} F^{-1^{k}}\left(B^{\perp}\right)=\lim _{k \rightarrow \infty}\left(F^{* k}(B)\right)^{\perp}=\left(\lim _{k \rightarrow \infty} F^{* k}(B)\right)^{\perp}=A^{\prime \perp} .
$$

Note that we have also shown that $B_{A^{\prime}}$ is the basin of attraction for $A^{\prime}$ of $F^{*}$ if and only if $\left(B_{A^{\prime}}\right)^{\perp}$ is the basin of attraction for $R$ of $F^{-1}$.

The implications $(3) \Rightarrow(1)$ can be obtained by replacing $F$ by $F^{-1}$ in the above arguments.

Concerning the statements about the basins of attraction, we will show that $B_{A}=\widehat{\mathbb{C}} \backslash R ; B_{R}=\widehat{\mathbb{C}} \backslash A$ is proved in exactly the same way with $F^{-1}$ replacing $F$. We first show that $B_{A} \cap R=\emptyset$. Let $X_{F}$ be the set of repelling fixed points of $F$, i.e., the set of attracting fixed points of $F^{-1}$, and let

$$
R^{\prime}=\overline{\bigcup_{k=1}^{\infty} F^{-k}\left(X_{F}\right)} .
$$

By Theorem 3.6, $B_{A} \cap X_{F}=\emptyset$. Therefore $B_{A} \cap F^{-k}\left(X_{F}\right)=\emptyset$ for $k \geq 1$; otherwise if $z \in B_{A} \cap F^{-k}\left(X_{F}\right)$, then for some $g \in F^{k}$ we would have $g(z) \in g\left(B_{A}\right) \cap$ $X_{F} \subseteq B_{A} \cap X_{F}$. We now have $B_{A} \cap \bigcup_{k=1}^{\infty} F^{-k}\left(X_{F}\right)=\emptyset$, and hence $B_{A} \cap R^{\prime}=\emptyset$ because $B_{A}$ is an open set. We claim that $R^{\prime}=R$, which would complete the proof that $B_{A} \cap R=\emptyset$. Concerning the claim, since $R$ is the attractor of $F^{-1}$, $R=\lim _{k \rightarrow \infty} F^{-k}\left(X_{f}\right) \subset R^{\prime}$. Since $X_{F} \subset R$, also $R^{\prime} \subset R$.

To finish the proof that $B_{A}=\widehat{\mathbb{C}} \backslash R$, notice that it has already been shown that $F^{*}$ has an attractor $A^{\prime} \neq \widehat{\mathbb{C}}$. According to Theorem 4.6, $A^{\prime}$ is contained in a set $U$ such that $F^{*}$ is a topological contractive on $\bar{U}$. With little change to the proof of Theorem 4.6. it follows that if $N$ is any open set containing $A^{\prime}$, then such a set $U$ can be chosen so that $\bar{U} \subset N$. So, for any $\epsilon>0$, let $A_{\epsilon}^{\prime}$ be a compact set containing $A^{\prime}$, contained in the $\epsilon$-neighborhood $\left\{z: d(z, w) \leq \epsilon\right.$ for some $\left.w \in A^{\prime}\right\}$ of $A^{\prime}$, and such that $F^{*}\left(A_{\epsilon}^{\prime}\right) \subset \operatorname{int}\left(A_{\epsilon}^{\prime}\right)$. This last inclusion implies, by Proposition 5.3, that

$$
F\left(\overline{A_{\epsilon}^{\prime *}}\right) \subset F\left(\left(\operatorname{int} A_{\epsilon}^{\prime}\right)^{*}\right) \subset A_{\epsilon}^{\prime *} .
$$

But according to Theorem 4.5, $F\left(\overline{A_{\epsilon}^{\prime *}}\right) \subset A_{\epsilon}^{\prime *}$ implies that $F$ is contractive on $A_{\epsilon}^{\prime *}$, and hence, by the classic result of Hutchinson [13], we have $A \subset A_{\epsilon}^{\prime *} \subset B_{A}$. Therefore

$$
R \subset \widehat{\mathbb{C}} \backslash B_{A} \subset \widehat{\mathbb{C}} \backslash A_{\epsilon}^{\prime *}=A_{\epsilon}^{\prime \perp} .
$$

Since $\lim _{\epsilon \rightarrow 0} A_{\epsilon}^{\prime}=A^{\prime}$, also $\lim _{\epsilon \rightarrow 0} A_{\epsilon}^{\prime \perp}=A^{\prime \perp}=R$. Therefore $\widehat{\mathbb{C}} \backslash B_{A}=R$ or $B_{A}=\widehat{\mathbb{C}} \backslash R$.

Having shown that $\left(B_{A^{\prime}}\right)^{\perp}=B_{R}$, it follows that $B_{A^{\prime}}=\left(B_{R}\right)^{\perp}=(\widehat{\mathbb{C}} \backslash A)^{\perp}$. 


\section{Chain-Recurrence}

In this section, after defining the notion of a chain-recurrent point, we prove the equivalence of statements (1) and (5) in Theorem 1.1 .

Definition 6.1. Let $F$ be an IFS on a metric space with metric $d(\cdot, \cdot)$. Given $\varepsilon>0$, an $\varepsilon$-chain for $F$ is a sequence of points $\left\{z_{i}\right\}_{i=0}^{n}, n>0$, in $\widehat{\mathbb{C}}$ such that, for each $i \in\{0,1,2, \ldots, n-1\}$, there is an $f \in F$ such that $d\left(z_{i+1}, f\left(z_{i}\right)\right)<\varepsilon$. A point $z \in \mathbb{C}$ is chain-recurrent for $F$ if for every $\varepsilon>0$ there is an $\varepsilon$-chain $\left\{z_{i}\right\}_{i=0}^{n}$ for $F$ such that $z_{0}=z_{n}=z$. The set of all chain recurrent points for $F$ is denoted by $\mathcal{R}(F)$.

Theorem 6.2. A Möbius IFS $F$ has an attractor $A \neq \widehat{\mathbb{C}}$ if and only if $\mathcal{R}(F) \neq \widehat{\mathbb{C}}$, in which case $\mathcal{R}(F)=A \cup R$, where $R$ is the repeller of $F$.

Proof. Assume $\mathcal{R}(F) \neq \widehat{\mathbb{C}}$. To prove that $A \neq \widehat{\mathbb{C}}$, let $z \notin \mathcal{R}(F)$. Then there is an $\varepsilon>0$ such that no $\varepsilon$-chain starts and ends at $z$. Let $U$ denote the set of all points $w$ such that there is an $\varepsilon$-chain from $z$ to $w$. Notice that (1) $z \notin U,(2) U$ is an open set, and $(3) F(\bar{U}) \subset U$. By the already proved implication $(3) \Rightarrow(1)$ in Theorem 1.1. the IFS $F$ has an attractor $A$ contained in $U$. Since $z \notin U$, we have $z \notin A$ and $A \neq \widehat{\mathbb{C}}$.

The paragraph above shows that if $z \notin \mathcal{R}(F)$, then $z \notin A$. Hence $A \subset \mathcal{R}(F)$. To show that $R \subset \mathcal{R}(F)$, let $z \in R$ and let $\varepsilon>0$. An $\varepsilon$-chain for $F$ starting and ending at $z$ is constructed as follows. By the continuity of the functions in $F$, there exists a $\delta>0$ such that, if $d_{c}(x, y)<\delta$, then $d_{c}(f(x), f(y))<\varepsilon$ for all $x, y \in \widehat{\mathbb{C}}$ and all $f \in F$. Since $\lim _{k \rightarrow \infty} F^{-k}(\{z\})=R$, there is sequence $f_{i_{1}}, f_{i_{2}}, \ldots, f_{i_{N}}$ of functions in $F$ such that $d_{c}\left(f_{i_{1}}^{-1} \circ f_{i_{2}}^{-1} \circ \cdots \circ f_{i_{N}}^{-1}(z), z\right)<\delta$. Let $w_{N}=z$ and $w_{N-1}=f_{i_{N}}^{-1}\left(w_{N}\right), w_{N-2}=f_{i_{N-1}}^{-1}\left(w_{N-1}\right), \ldots, w_{0}=f_{i_{1}}^{-1}\left(w_{1}\right)$. Further, let $z_{0}=z$ and $z_{k}=w_{k}$ for $k=1,2, \ldots, N$. Since $d_{c}\left(z_{0}, w_{0}\right)<\delta$, we have $d_{c}\left(f_{i_{1}}\left(z_{0}\right), z_{1}\right)=d_{c}\left(f_{i_{1}}\left(z_{0}\right), f_{i_{1}}\left(w_{0}\right)\right)<\varepsilon$. Also $d_{c}\left(f_{i_{k}}\left(z_{k-1}\right), z_{k}\right)=d_{c}\left(w_{k}, w_{k}\right)=0$ for $k=2,3, \ldots, N$. We have shown that $z$ is chain-recurrent, hence $A \cup R \subset \mathcal{R}(F)$.

Now assume that $F$ has an attractor $A \neq \widehat{\mathbb{C}}$. By Theorem 5.4 , the IFS $F$ has a unique repeller $R$ and $A \cap R=\emptyset$. Since $A$ and $R$ are compact, $A \cap R \neq \widehat{\mathbb{C}}$. To finish the proof it suffices to show that $\mathcal{R}(F)=A \cup R$. We already have $A \cup R \subseteq \mathcal{R}(F)$. To show that $\mathcal{R}(F) \subseteq A \cup R$, let $z \notin A \cup R$. We will show that $z$ is not chainrecurrent. Since $z \notin R$, by Theorem 5.4 the point $z$ lies in the basin of attraction of $A$, and hence $\lim _{k \rightarrow \infty} F^{n}(\{z\}) \in A$. Let $U^{\prime} \subset U$ be two open sets containing $A$ such that $\inf _{x \in \bar{U}^{\prime}, y \in \widehat{\mathbb{C} \backslash U}} d_{c}(x, y)>0$. Since $\lim _{k \rightarrow \infty} F^{k}(\{z\}) \in A$ there is a $K$ such that $F^{k}(\{z\}) \subset U^{\prime}$ for all $k \geq K$ and, by the continuity of the functions in $F$, an $\varepsilon(k)>0$ such that every $\varepsilon$-chain of length $k \geq K$ starting at $z$ ends in $U$. If there is an $\varepsilon$ chain starting and ending at $z$, then there is, by repeating the chain if necessary, an $\varepsilon$ chain starting and ending at $z$ of length at least $K$. But we have shown that it cannot be the case that, for every $\varepsilon$, there is an $\varepsilon$-chain starting and ending at $z$ of length at least $K$. Therefore $z$ is not chain-recurrent.

\section{EXAmples}

Example 7.1. If $F=(\widehat{\mathbb{C}} ; f)$ consists of a single loxodromic function $f$, then the attractor is the attracting fixed point of $f$ and the repeller is the repelling fixed point of $f$. 
If a Möbius IFS $F$ has an attractor, not equal to $\widehat{\mathbb{C}}$, then by Theorem 3.6 each transformation in $F$ is loxodromic. A general loxodromic transformation $f$ can be expressed as $f=g^{-1} \cdot \hat{f} \cdot g$, where $g$ is an arbitrary Möbius transformation and $\hat{f}(z)=s z$, where $s \in \mathbb{C},|s|<1$. The contractivity parameter $s$ gives an indication of how attractive is the fixed point 0 of $\hat{f}$, and hence how attractive is the the attracting fixed point of $f$. Basically, the smaller the modulus $|s|$, the more attractive. Letting $g(z)=(A z+B) /(C z+D)$, the fixed points of $f$ are $-B / A$ and $-D / C$. A straightforward calculation then suffices to expess a general loxodromic transformation in terms of three parameters, the attracting fixed point $a$, the repelling fixed point $r$, and $s$. If $a \neq \infty$ and $r \neq \infty$, then $f$ has the form:

$$
f(z)=\frac{(a-s r) z+(s-1) a r}{(1-s) z+(s a-r)},
$$

where $1>|s| \geq 0$, If $r=\infty$, the functon takes the form $f(z)=s z+a(1-s)$. If $s=0$, then $f(z)=a$, and if $s=1$, then $f(z)=z$. If the parameter $s$ is real, then the loxodromic transformation $f$ is called hyperbolic.

Example 7.2. Figure 3 shows the attractor and repeller of an IFS $\left\{\widehat{\mathbb{C}} ; f_{1}, f_{2}, f_{3}\right\}$, where the three attractive fixed points $x_{1}, x_{2}, x_{3}$, respectively, of the three hyperbolic functions are mutually orthogonal as vectors on the Riemann sphere. The repelling fixed points $x_{1}^{\prime}, x_{2}^{\prime}, x_{3}^{\prime}$ are diametrically opposite the points $x_{1}, x_{2}, x_{3}$, respectively. The contractivity factor $s$ in (7.1) is 0.4. In Figure 4 the IFS is basically the same IFS except that $s=.4 e^{.4 i}$ (not hyperbolic).

Example 7.3. Figure 5 shows the attractor of the IFS consisting of four loxodromic functions whose attracting fixed points are located at the four vertices of a regular tetrahedron inscribed in the Riemann sphere. The repelling fixed points are located diametrically opposite the respective fixed points. The factor $s=.13 e^{.5 i}$.

Example 7.4. Figure 1 in the introduction shows the attractor and repeller of the IFS $F=\left(\widehat{\mathbb{C}} ; f_{1}, f_{2}\right)$ where

$$
\begin{aligned}
L_{f_{1}} & =\left(\begin{array}{cc}
0.1566-0.4101 i & 0.4453-0.3560 i \\
0.4305-0.3896 i & -0.7477-1.2362 i
\end{array}\right), \\
L_{f_{2}} & =\left(\begin{array}{ll}
1.0122-0.0984 i & 0.3985-0.4076 i \\
0.4305-0.3896 i & 0.2801-1.0804 i
\end{array}\right) .
\end{aligned}
$$

\section{LORENZ TRANSFORMATIONS}

In addition to the three equivalent viewpoints given in section 2 regarding a Möbius transformation, there is a fourth - as a Lorentz transformation on the celestial sphere in Minkowski space. This is the perspective taken in [14, 15], where the corresponding iterated function system is referred to as a "quantum iterated function system".

Minkowski space consists of $\mathbb{R}^{4}$ together with the quadratic form

$$
Q(\mathbf{x})=Q\left(x_{0}, x_{1}, x_{2}, x_{3}\right)=x_{0}^{2}-x_{1}^{2}-x_{2}^{2}-x_{3}^{2} .
$$

The future light cone $\mathrm{N}^{+}$is defined as

$$
N^{+}=\left\{\mathbf{x}=\left(x_{0}, x_{1}, x_{2}, x_{3}\right) \in \mathbb{R}^{4}: x_{0}>0, Q(\mathbf{x})=0\right\},
$$



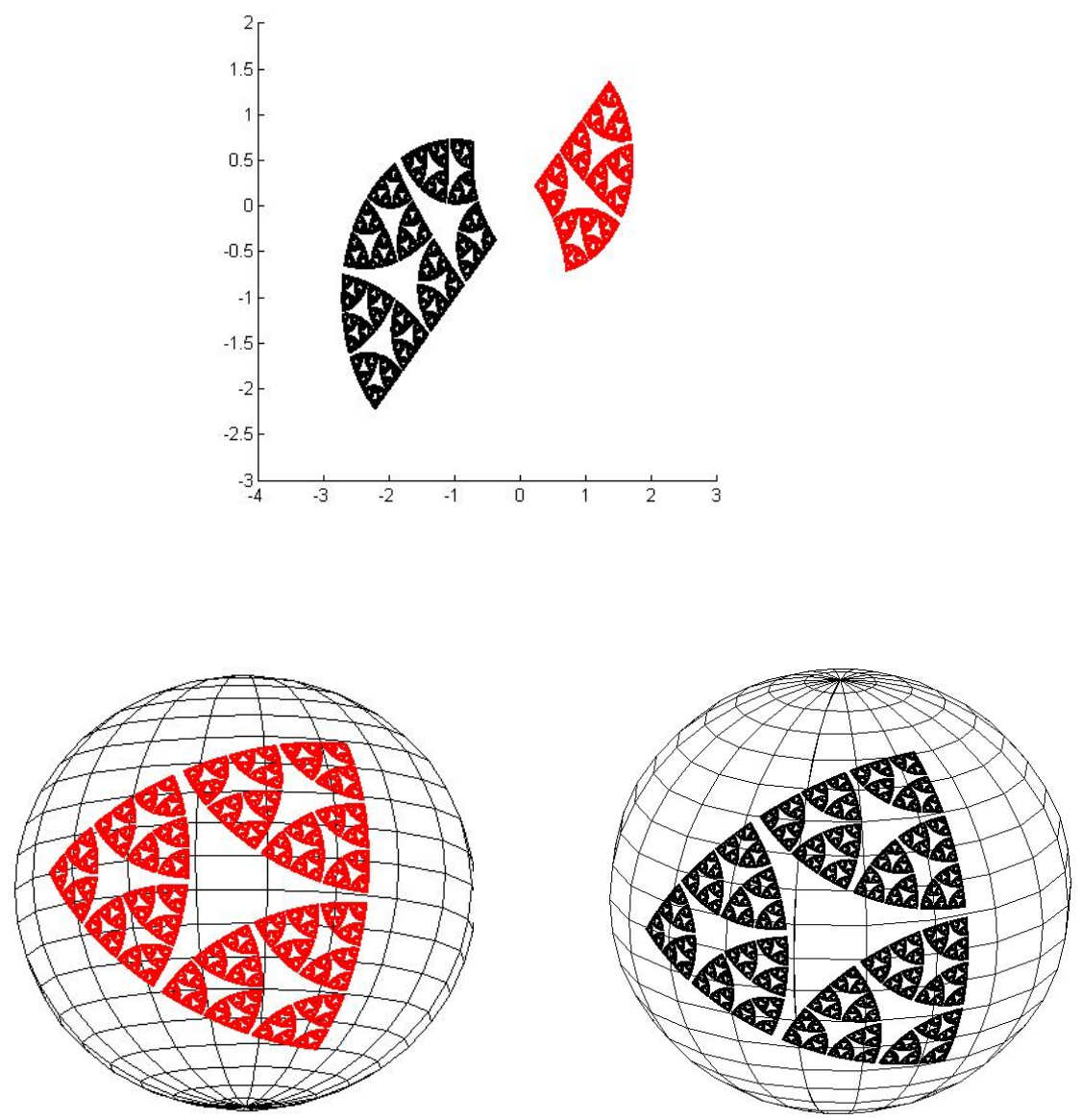

FiguRE 3. The attractor (red, right) and repeller (black, left) of Example 7.2 are depicted in the complex plane (top figure), and on opposite sides of the Riemann sphere (bottom figure-attractor on the left, repeller on the right). See the online version for colors.

and the celestial sphere $\mathcal{C}$ is the set of rays in $N^{+}$whose initial point is the origin of $\mathbb{R}^{4}$. The celestial sphere can thus be regarded as the projectivized future light cone: $\mathcal{C}=\mathbb{P} N^{+}$. The restricted Lorentz group $S O^{+}(1,3)$ is the set of linear transformations of $\mathbb{R}^{4}$ with positive determinant that preserves the quadratic form $Q$ and preserves the time direction, i.e., $\mathbf{x}$ is future pointing if $x_{0}>0$. Therefore the restricted Lorentz group acts on the celestial sphere.

There is a bijection

$$
\begin{aligned}
\mathbb{C P}^{1} & \rightarrow \mathcal{C}, \\
(z, w) & \mapsto\left(x_{0}, x_{1}, x_{2}, x_{3}\right),
\end{aligned}
$$




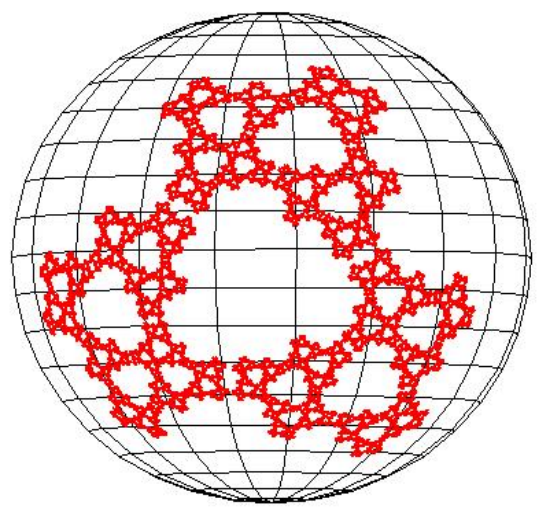

FiguRE 4. The attractor for the second IFS in Example 7.2

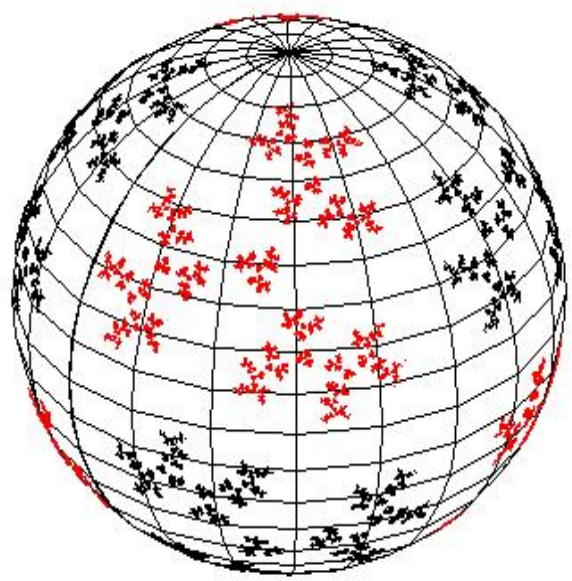

Figure 5. The attractor (red) and repeller (black) in Example 7.3. See the online version for colors. 
defined by

$$
2\left(\begin{array}{c}
z \\
w
\end{array}\right)\left(\begin{array}{ll}
z & w
\end{array}\right)=X:=\left(\begin{array}{cc}
x_{0}+x_{1} & x_{2}+i x_{3} \\
x_{2}-i x_{3} & x_{0}-x_{1}
\end{array}\right) .
$$

A projective transformation $L: \mathbb{C} \mathbf{P}^{1} \rightarrow \mathbb{C P}^{1}$ corresponds to the Lorentz transformation

$$
\begin{aligned}
\mathcal{C} & \rightarrow \mathcal{C}, \\
X & \mapsto L X L^{*},
\end{aligned}
$$

where $L^{*}$ is the conjugate transpose matrix.

By letting $\mathbb{S}^{+}$be the intersection of $N^{+}$with the hyperplane $x_{0}=1$ in $\mathbb{R}^{4}$, the celestial sphere $\mathcal{C}$ may be identified with the sphere $\mathbb{S}^{+}=\left\{\left(x_{1}, x_{2}, x_{3}\right): x_{1}^{1}+x_{2}^{2}+\right.$ $\left.x_{3}^{2}=1\right\}$ in the 3 -dimensional subspace of $\mathbb{R}^{4}$ spanned by the $x_{1}, x_{2}$ and $x_{3}$ axes. A transformation in $S O^{+}(1,3)$ does not necessarily take the hyperplane $x_{0}=1$ to itself, but if we multiply by a suitable scalar so that $x_{0}=1$, then the Lorentz group can be regarded as acting on $\mathbb{S}^{+}$. This turns out to be precisely the action described in section 2 of $P S L(2, \mathbb{C})$ on the Riemann sphere.

\section{Strongly LOXODROMiC IFSs}

In this last section we introduce a property of a Möbius IFS that we conjecture is equivalent to the other five conditions in Theorem 1.1. Representing a Möbius transformation $f$ by a $2 \times 2$ matrix $L_{f}$ with eigenvalues $\lambda_{1}(f), \lambda_{2}(f)$ with $\left|\lambda_{1}(f)\right| \leq$ $\left|\lambda_{2}(f)\right|$, let

$$
\lambda(f):=\left|\frac{\lambda_{1}(f)}{\lambda_{2}(f)}\right| \leq 1 .
$$

Let $\Omega_{k}(F)$ denote the set of all $k$-fold compositions of functions in $F$ and let

$$
\begin{aligned}
M_{k}(F) & =\max _{f \in \Omega_{k}(F)} \lambda(f), \\
\lambda(F) & =\limsup _{k \rightarrow \infty}\left[M_{k}(F)\right]^{1 / k} .
\end{aligned}
$$

Call a Möbius IFS F strongly loxodromic if

$$
\lambda(F)<1 .
$$

A Möbius transformation $f$ is parabolic or elliptic if and only if $\lambda(f)=1$. Therefore a strongly loxodromic IFS is loxodromic.

Conjecture 9.1. A Möbius IFS $F$ is strongly loxodromic if and only if $\mathcal{R}(F) \neq \widehat{\mathbb{C}}$.

\section{REFERENCES}

[1] Ross Atkins, M. F. Barnsley, David C. Wilson, Andrew Vince, A characterization of pointfibred affine iterated function systems, Topology Proceedings 38 (2010) 189-211. MR2609349 (2011f:54060)

[2] M. F. Barnsley and A. Vince, Real projective iterated function systems, J. Geometric Analysis, to appear.

[3] M. F. Barnsley, Fractals Everywhere, Academic Press, Boston, MA, 1988. MR.1231795 (94h:58101)

[4] M. F. Barnsley, Fractal image compression, Notices Amer. Math. Soc. 43 (1996) 657-662. MR 1388729

[5] M. F. Barnsley, J. Hutchinson, Ö. Stenflo, V-variable fractals: fractals with partial self similarity, Advances in Mathematics, 218 (2008) 2051-2088. MR2431670 (2010g:28010)

[6] M. A. Berger and Y. Wang, Bounded semigroups of matrices, Linear Algebra and Appl. 166 (1992), 21-27. MR1152485 (92m:15012) 
[7] J. Blanc-Talon, Self-controlled fractal splines for terrain reconstruction, IMACS World Congress on Scientific Computation, Modelling, and Applied Mathematics 114 (1997), 185-204.

[8] L. Dubois, Projective metrics and contraction principles for complex cones, J. London Math. Soc. 79 (2009) 719-737. MR2506695 (2010g:47160)

[9] Kenneth Falconer, Fractal Geometry: Mathematical Foundations and Applications, John Wiley and Sons, Ltd., Chichester, 1990. MR.1102677 (92j:28008)

[10] Yuval Fisher, Fractal Image Compression: Theory and Application, Springer Verlag, New York, 1995. MR1313035 (95k:68237)

[11] P. Jorgensen, Analysis and Probability: Waveletes, Signals, Fractals, Springer, New York, 2006. MR2254502(2008a:42030)

[12] D. Hilbert, Über die gerade Linie als kurseste Verbindung zweier Punkte, Math., Ann. 46 (1985) 91-96.

[13] J. Hutchinson, Fractals and self-similarity, Indiana Univ. Math. J. 30 (1981) 713-747. MR625600(82h:49026)

[14] A. Jadczyk, On quantum iterated function systems, Central Europ. J. Physics, 2 (2004) 492-503.

[15] A. Łozińsky, K. Życzkowski, and W. Słomezyński, Quantum iterated function systems, Physical Review E, 68 (2003) 046110. MR2060853(2005a:82065)

[16] D. Mumford, C. Series and D. Wright, Indra's Pearls, the Vision of Felix Klein, Cambridge University Press, Cambridge, United Kingdom, 2002. MR 1913879 (2003f:00005)

Department of Mathematics, University of Florida, Gainesville, Florida 32611

E-mail address: avince@ufl.edu 